\title{
Development of a high-performance cryogenic shock tube
}

\author{
By JOHN C. CUMMINGS \\ Graduate Aeronautical Laboratories, California Institute of Technology, Pasadena $\dagger$
}

(Received 19 February 1974)

A cryogenic shock tube has been developed as a tool for research in fluid mechanics and low-temperature physics. The shock tube was designed to operate with the test section immersed in a cryogenic liquid. A unique diaphragm-changing mechanism makes this shock tube an economical and practical device. There are several advantages in operating a shock tube at cryogenic temperatures. Shock waves of very large Mach number can be produced. The flow field can be accurately calculated using ideal-shock-tube/perfect-gas theory. Boundary-layer effects are decreased, so that long test times are possible.

The cases that were studied are test-gas temperatures of $300,77,4 \cdot 2$ and $2 \cdot 3{ }^{\circ} \mathrm{K}$. Helium was used as both test and driver gas. The largest Mach numbers observed range from 2.4 at $300^{\circ} \mathrm{K}$ to 32 at $2 \cdot 3^{\circ} \mathrm{K}$ (several runs at $1.46^{\circ} \mathrm{K}$ produced Mach 40 shocks). As the temperature of the test gas was decreased, the observed Mach numbers approached those calculated using the 'shock-tube equation'.

As a laboratory tool, the cryogenic shock tube may be applied in many areas and modified for use in even more. Shock waves with large Mach numbers and flows with large Reynolds numbers can be produced with this device. The rapid increase in temperature and pressure across the shock wave is useful for studies of sublimation, evaporation or chemical reactions. Quantum-mechanical effects in cryogenic materials, superconductors or superfluid helium can also be investigated.

\section{Introduction}

In the field of fluid mechanics the advantages of working at cryogenic temperatures have been demonstrated in the development of a low-temperature ballistics range (Bennett 1957), a cryogenic wind tunnel (Goodyer \& Kilgore 1972) and two cryogenic shock tubes (Yoder 1971; Rupert 1972). The present work discusses the development and characterization of a unique cryogenic shock tube which has produced shock waves with large Mach numbers $(\sim 40)$ governed by ideal-shock-tube/perfect-gas equations. $\ddagger$

Since its initial use over seventy years ago (Vielle 1899) the shock tube has

† Present address: Fluid Mechanies Laboratory, TRW Systems, Redondo Beach, California 90278.

$\mp$ Preliminary results have been presented elsewhere (Liepmann, Cummings \& Rupert 1973 
evolved into an extremely valuable tool in the fluid-mechanics laboratory. Much of the effort to obtain shock waves with large Mach numbers has been directed towards techniques to increase the temperature and pressure of the driver gas. Conventional, electromagnetic, detonation and wave-process methods are the general techniques used with high-performance driver sections that can generate shock waves with Mach numbers of 60 and larger (Warren \& Harris 1969). Testsection modifications to increase shock Mach numbers consist mainly of geometrical convergences. From the analysis that follows, it will be clear that a shock tube that operates with the test gas at cryogenic temperatures can, under certain conditions, produce shock waves with very large Mach numbers and with accurately known flow conditions in all shock-tube regions.

Of great importance to the present work was the need for 'diaphragm temperature ratios' $T_{4} / T_{1}$ of 100 or larger. As an engineering problem, it was necessary to limit the extent of gas temperature gradients to not more than several tube diameters upstream and downstream of the diaphragm location. A novel diaphragm-changing mechanism has been developed which makes this shock tube a practical laboratory device. The new shock tube is viewed as a prototype tool for the study of both fluid-mechanical and cryogenic phenomena.

\section{Basic principles of a cryogenic shock tube}

\subsection{Large Mach numbers}

The Mach number of a shock wave in an ideal shock tube (containing perfect gases in all flow regions) is related to the diaphragm pressure ratio $p_{4} / p_{1}$, the ratio of the sound speed in the driver to that in the test gas and the ratio of specific heats $\gamma$ for each gas. 'This relation is commonly known as the 'shock-tube equation':

$$
\frac{p_{4}}{p_{1}}=\frac{1+2 \gamma_{1}\left(\gamma_{1}+1\right)^{-1}\left(M_{s}^{2}-1\right)}{\left[1-\frac{\gamma_{4}-1}{\gamma_{1}+1} \frac{a_{1}}{a_{4}} \frac{M_{s}^{2}-1}{M_{s}}\right]^{2 \gamma_{4} /\left(\gamma_{4}-1\right)}},
$$

where $M_{s}$ is the shock-wave Mach number, $a$ the speed of sound and the subscripts 1 and 4 refer to the undisturbed test gas and undisturbed driver gas respectively. Equation (1) indicates that it is possible to increase the shock Mach number by increasing the ratio of the speeds of sound $a_{4} / a_{1}$. 'High-performance' shock tubes increase the temperature of the driver gas in order to raise the driver-gas sound speed. A cryogenic shock tube can lower the test-gas sound speed by decreasing the temperature of the test gas.

Note that as the diaphragm pressure ratio approaches infinity the shock Mach number approaches a limiting value $\widehat{M}_{s}$, where

$$
\widehat{M}_{s}=\frac{1}{2} \frac{\gamma_{1}+1}{\gamma_{4}-1} \frac{a_{4}}{a_{1}}+\left[\left(\frac{1}{2} \frac{\gamma_{1}+1}{\gamma_{4}-1} \frac{a_{4}}{a_{1}}\right)^{2}+1\right]^{\frac{1}{2}}
$$

Consider the special case where helium is used as driver and test gas and assume that the helium behaves as a perfect gas. The limiting Mach number takes the form

$$
\widehat{M}_{s}=2\left(\frac{T_{4}}{T_{1}}\right)^{\frac{1}{2}}+2\left(\frac{T_{4}}{T_{1}}+\frac{1}{4}\right)^{\frac{1}{2}}
$$


and the increase in Mach number with increasing $T_{4}$ or decreasing $T_{1}$ is more evident. To compare the two types of temperature variation it is convenient to consider $T_{4} / T_{1} \gg \frac{1}{4}$. Then ( 3 ) reduces to

$$
\widehat{M}_{s} \doteq 4\left(T_{4} / T_{1}\right)^{\frac{1}{2}}
$$

Equation (4) illustrates the advantage of attaining high Mach numbers with cryogenic temperatures. For a limiting Mach number of 40 the diaphragm temperature ratio must be 100 . The high-performance-driver (HPD) shock tubes generally operate with $T_{1}=300^{\circ} \mathrm{K}$, hence $T_{4}$ must approach $30000^{\circ} \mathrm{K}$ to obtain shock Mach numbers near 40 . If a cryogenic shock tube operates with $T_{4}=300^{\circ} \mathrm{K}$, the test gas must be cooled to $3^{\circ} \mathrm{K}$ to achieve the same Mach number.

\subsection{Boundary-layer effects}

Boundary-layer growth behind the shock wave and expansion fan causes deceleration of the shock, two-dimensional effects and a limitation on the test time. Roshko (1960) and Mirels (1963) developed a laminar boundary-layer theory capable of estimating the effect on the test time. The corresponding case for a turbulent boundary layer was analysed by Mirels (1964). The theories indicate that the maximum test time $\tau_{m}$ can be written as

$$
\tau_{m}=\left\{\begin{array}{ll}
F_{L}\left(\gamma_{1}, M_{s}\right)\left[d \rho_{1} / \mu_{w}\right] d & \text { for a laminar boundary layer, } \\
F_{T}\left(\gamma_{1}, M_{s}\right)\left[d \rho_{1} / a_{1}^{3} \mu_{w}\right]^{\frac{1}{4}} d & \text { for a turbulent boundary layer, }
\end{array}\right\}
$$

where $d$ is the diameter of the shock tube and $\mu_{w}$ is the viscosity of the gas at the wall temperature.

As an extension of the comparison between the HPD shock tubes and a cryogenic tube it is instructive to rewrite (5) for the two types of shock tube. If the test gas, Mach number, test pressure and tube diameter are similar, then

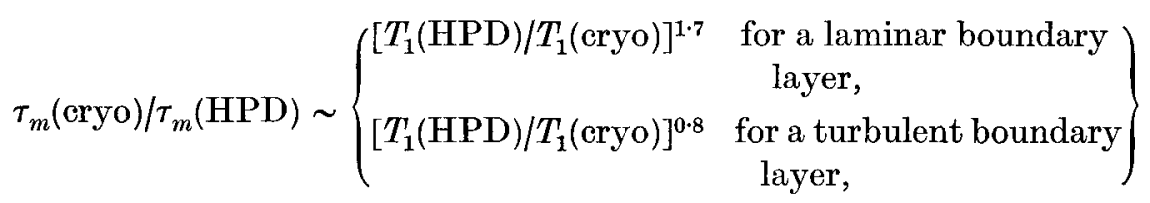

where the viscosity of the test gas is determined as $\mu_{w} \doteq \mu_{0}\left(T_{1} / T_{0}\right)^{0 \cdot 7}$, the subscript zero denoting conditions at room temperature. Equation (6) indicates that, if the cryogenic tube uses a test gas at a temperature $T_{1}$ (cryo) $=3^{\circ} \mathrm{K}$, and if the HPD tube operates with a test gas at room temperature $\left(T_{1}(\mathrm{HPD})=300^{\circ} \mathrm{K}\right)$, then the limiting test time for the cryogenic tube can be a factor of a thousand larger than that for the HPD tube.

An important implication of (5) is that acceptable test times can be obtained using a test section of small diameter in a cryogenic shock tube.

\subsection{Ideal-gas behaviour}

The temperature range of ideal-gas behaviour is larger for helium than for any other gas. The temperature of helium gas is not 'high' until it approaches $285000^{\circ} \mathrm{K}$ (the ionization temperature $\theta_{i}$ ) and it is not 'low' until it approaches 
$5 \cdot 2^{\circ} \mathrm{K}$ (the critical temperature $T_{c}$ ). If the temperature in any region of the shock tube is of the order of $T_{\mathrm{c}}$ or $\theta_{i}$, the pressure of the gas in that region will determine the 'idealness'.

For an ideal shock tube using ideal gases it is possible to consider real-gas effects in the light of a worst-case analysis. Consider the limiting case when $p_{4} / p_{1} \rightarrow \infty$ and $T_{4} / T_{1} \gg 1$. For this condition it can be shown that

$$
\left.\begin{array}{rl}
p_{2} / p_{1} \leqslant 20\left(T_{4} / T_{1}\right), & p_{5} / p_{1} \leqslant 120\left(T_{4} / T_{1}\right), \\
T_{2} \leqslant 5 T_{4}, & T_{5} \leqslant 12 T_{4},
\end{array}\right\}
$$

where the subscripts 2 and 5 refer to the test gas behind the incident and reflected shock respectively. Equations (7) indicate the dependence of flow quantities on the diaphragm temperature ratio. More important, for a cryogenic shock tube operated with $T_{4} \sim 300^{\circ} \mathrm{K}$, is the limitation on the gas temperatures behind both the incident and reflected shocks to less than $3600^{\circ} \mathrm{K}$. Consequently real-gas effects (radiation, dissociation and ionization) can be negligible for a cryogenic shock tube, even for very strong shock waves.

Compressibility effects in a cryogenic shock tube are of concern regarding the idealness of the undisturbed test gas. For helium gas at $T_{1}=2 \cdot 3^{\circ} \mathrm{K}\left(T_{1} / T_{c}=0 \cdot 44\right)$ an analysis of the second virial coefficient $\uparrow$ indicates that the perfect-gas equation of state will be accurate to within $1 \%$ for $p_{1} \leqslant p_{s v} \ll p_{c}\left(p_{c}\right.$ is the critical pressure and $p_{s v}$ is the saturated vapour pressure).

As a further consideration of compressibility effects, it is necessary to investigate the possibility of condensation at the walls in flow regions behind the incident shock. In these regions the pressure may be above $p_{s v}$, and hence gas may condense at the walls. The extremely small heat capacity of the shock-tube wall at cryogenic tempera tures ensures a relatively large rise in wall temperature which prohibits gas condensation under certain conditions.

\section{Experimental apparatus and procedure}

\subsection{Shock-tube system}

A schematic diagram of the device is shown in figure 1. The shock tube consists of three stainless-steel tubes: the support tube, driver section and test section. The driver and test tubes have inner diameters of $1 \mathrm{in}$. and the test section is $22 \mathrm{in}$. long. The shock tube is mounted vertically with the test section at the lower end. The support tube serves the dual function of holding the test section rigidly in place and housing the driver section.

The driver section is made of two concentric tubes (figure 2). Radiation shielding was wrapped around the outside of each tube and a vacuum was drawn in the space between the tubes. In order to keep the driver-section inner tube at $\sim 300^{\circ} \mathrm{K}$, an electrical heating tape was wrapped around this tube.

A bellows-and-clamp arrangement permits the driver section to be displaced vertically while the entire shock tube is evacuated or filled with test gas. This

$\dagger$ Using a Lennard-Jones (6-12) potential and considering quantum-mechanical effects (Hirschfelder, Curtiss \& Bird 1964, ohap. 3). 


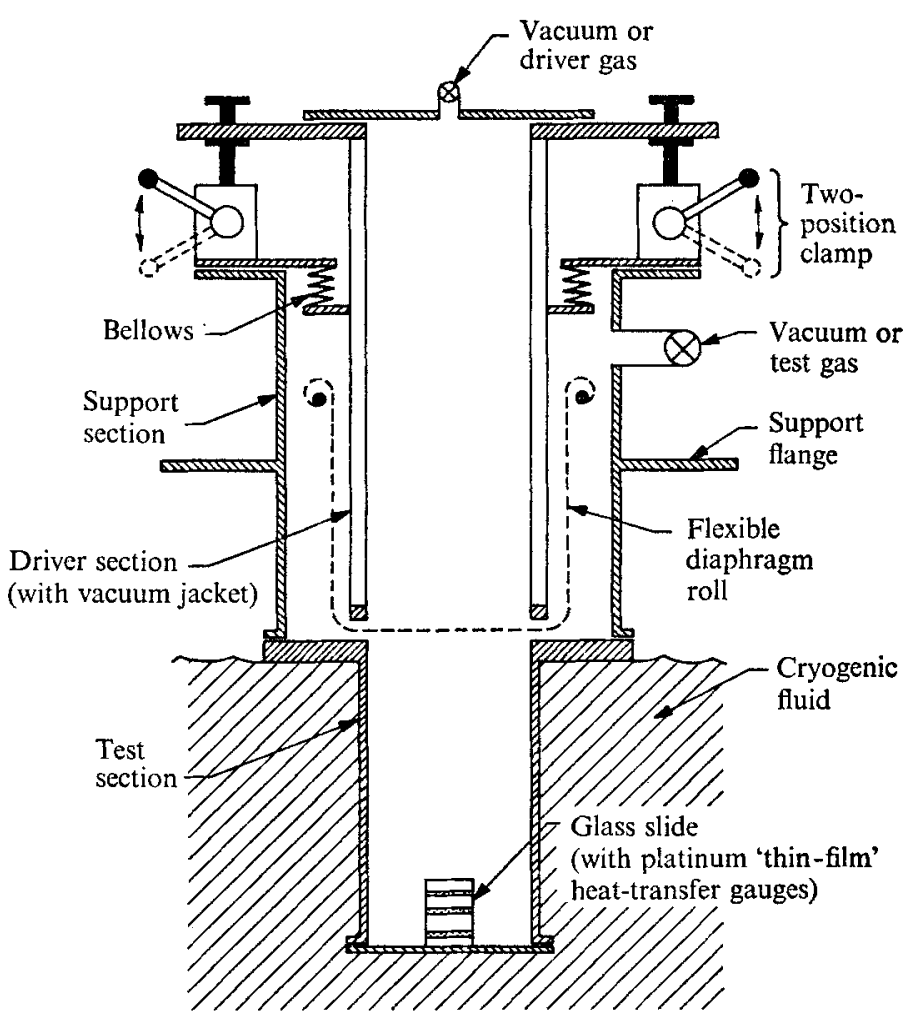

Fraure 1. Schematic drawing of the cryogenic shock tube.

allows the diaphragm to be changed in an uncontaminated environment (driver section lifted off the test section) and then allows the unruptured diaphragm to be sealed into position above the knife edges (driver section clamped down onto the test section).

The diaphragm-changing mechanism is the main design feature making the shock tube practical. $\dagger$ There is a direct analogy between this mechanism and that of a simple box camera. Starting from an output spindle the diaphragm runs vertically down the support tube, then between the driver and test sections, and finally up to a take-up spindle. This take-up spindle is attached to a roomtemperature region of the support tube and can be rotated manually from outside the tube.

The diaphragm material was a 2 in. wide roll of 0.0005 in. thick mylar. With a $20 \mathrm{ft}$ roll of diaphragm it was possible to complete 70 runs without reloading. When liquid helium was used as the test-section coolant, approximately 251 were required for these runs (with a corresponding cost per run of about $\$ 1$ ).

\subsection{Cryogenic system}

The shock tube was inserted into a vacuum-jacketed Dewar for runs at cryogenic temperatures. Liquified gas was transferred into the Dewar until the test section

$\dagger$ This was suggested by Prof. D. Coles of the California Institute of Technology. 


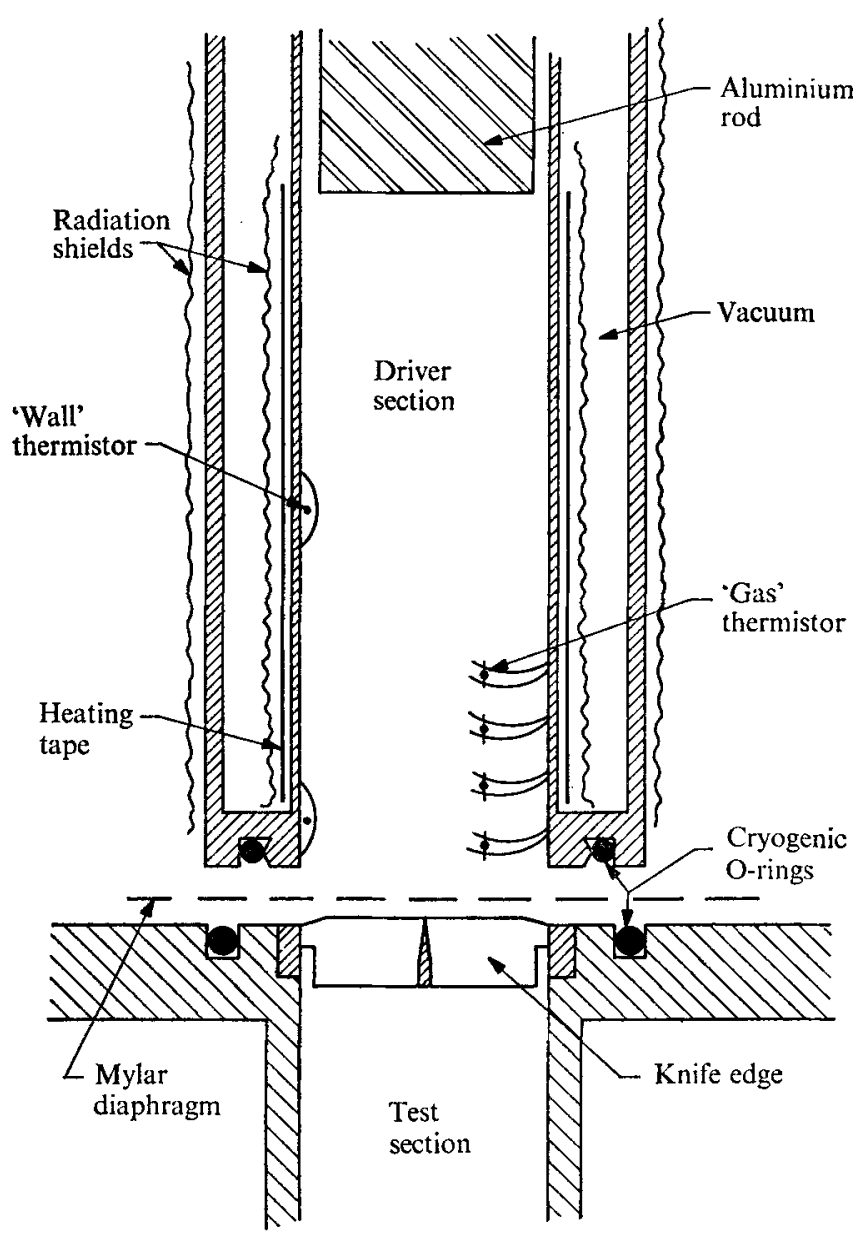

FIGURE 2. Details of the driver and test sections.

was fully immersed. A stainless-steel Dewar was used with liquid nitrogen and a silvered-Pyrex Dewar with liquid helium. The pressure in the Pyrex Dewar could be regulated to control the liquid-helium bath temperature.

\subsection{Instrumentation}

When helium is used as both the driver and driven gas, it is possible to write the ideal shock velocity as

$$
U_{s}=U_{s}\left(T_{1}, T_{4} / T_{1}, p_{4} / p_{1}\right)
$$

The instrumentation was designed to measure or allow calculation of all of these quantities so that a comparison with the ideal value could be made.

The test pressure was monitored with an electronic manometer accurate to $\pm 2 \%$. A combination gauge was used to determine the diaphragm burst pressure $p_{4}$. The accuracy of this gauge reading was not better than $\pm 10 \%$, which was roughly the actual scatter in burst pressures over a day's runs.

The temperature of the cryogenic bath was determined from the saturated 
vapour pressure above the bath. On the basis of vapour-pressure/temperature scales the largest error in absolute temperature was $\pm 1 \%$. It was assumed that the temperature of the test gas was equal to the bath temperature. This assumption was verified for the liquid-nitrogen cases using a fine-wire thermocouple. Glass-bead thermistors were used in the driver section (figure 2) to determine both the wall temperature (so that the power dissipated by the heating tape could be regulated) and the driver-gas temperature (as a function of time) with an accuracy of $\pm 2 \%$.

Platinum 'thin-film' heat-transfer gauges were used in the determination of shock velocities and test times. The platinum films were baked onto a glass microscope slide. This was mounted on the test-section end flange so that the films were perpendicular to the axis of the shock tube. The leading edge of the slide was sharpened to reduce interference effects. The top film was used to trigger an oscilloscope which then recorded the arrival of the shock wave at the two lower films. The heat dissipated by the three films was varied between $10^{-3}$ and $4 \times 10^{-5} \mathrm{~W}$ (changing this value produced no effect on the shock velocity).

Distances between the films were measured with less than $\pm 0.2 \%$ error on an optical comparator, but errors in arrival time varied from $\pm 1 \%$ to $\pm 4 \%$ depending on the time increment, signal-to-noise ratio and signal rise time.

\section{Results and discussion}

Four cases were run in order to determine the performance characteristics of the cryogenic shock tube. Each case was defined by the temperature of the test gas, and within each case only the pressure of the test gas was varied. The four cases were

(a) $T_{1}=300^{\circ} \mathrm{K}$ (room temperature);

(b) $T_{1}=77 \cdot 36 \pm 0 \cdot 18^{\circ} \mathrm{K}$ (liquid nitrogen at $744 \mathrm{Torr}$ with a bath depth of $22 \mathrm{in}$.), referred to as $T_{1}=77^{\circ} \mathrm{K}$;

(c) $T_{1}=4 \cdot 196 \pm 0 \cdot 004^{\circ} \mathrm{K}$ (liquid helium at 744 Torr with an average bath depth of $20 \mathrm{in}$.), referred to as $T_{1}=4 \cdot 2^{\circ} \mathrm{K}$;

(d) $T_{1}=2 \cdot 287 \pm 0.023^{\circ} \mathrm{K}$ (liquid helium at 47 Torr with an average bath depth of $18 \mathrm{in}$.), referred to as $T_{1}=2 \cdot 3^{\circ} \mathrm{K}$.

A few runs were made with $T_{1}=1 \cdot 46^{\circ} \mathrm{K}$ (liquid helium at 3 Torr). Helium was used as both driver and test gas for all cases. The test-gas temperatures listed above represent the temperatures of the cryogenic bath at the half-depth. Surface $(-)$ and full-depth $(+)$ temperatures are also given.

\subsection{Measurements of shock-wave Mach numbers}

The main objective in characterizing the performance of the shock tube was the determination of shock-wave velocities. The observed shock velocity was used to compute the Mach number of the shock wave, which is a measure of merit for high-performance shock tubes. A comparison between observed and calculated shock Mach numbers is made in figure 3.

(a) $T_{1}=300^{\circ} \mathrm{K}$. The room-temperature results are presented in figure $3(a)$ 

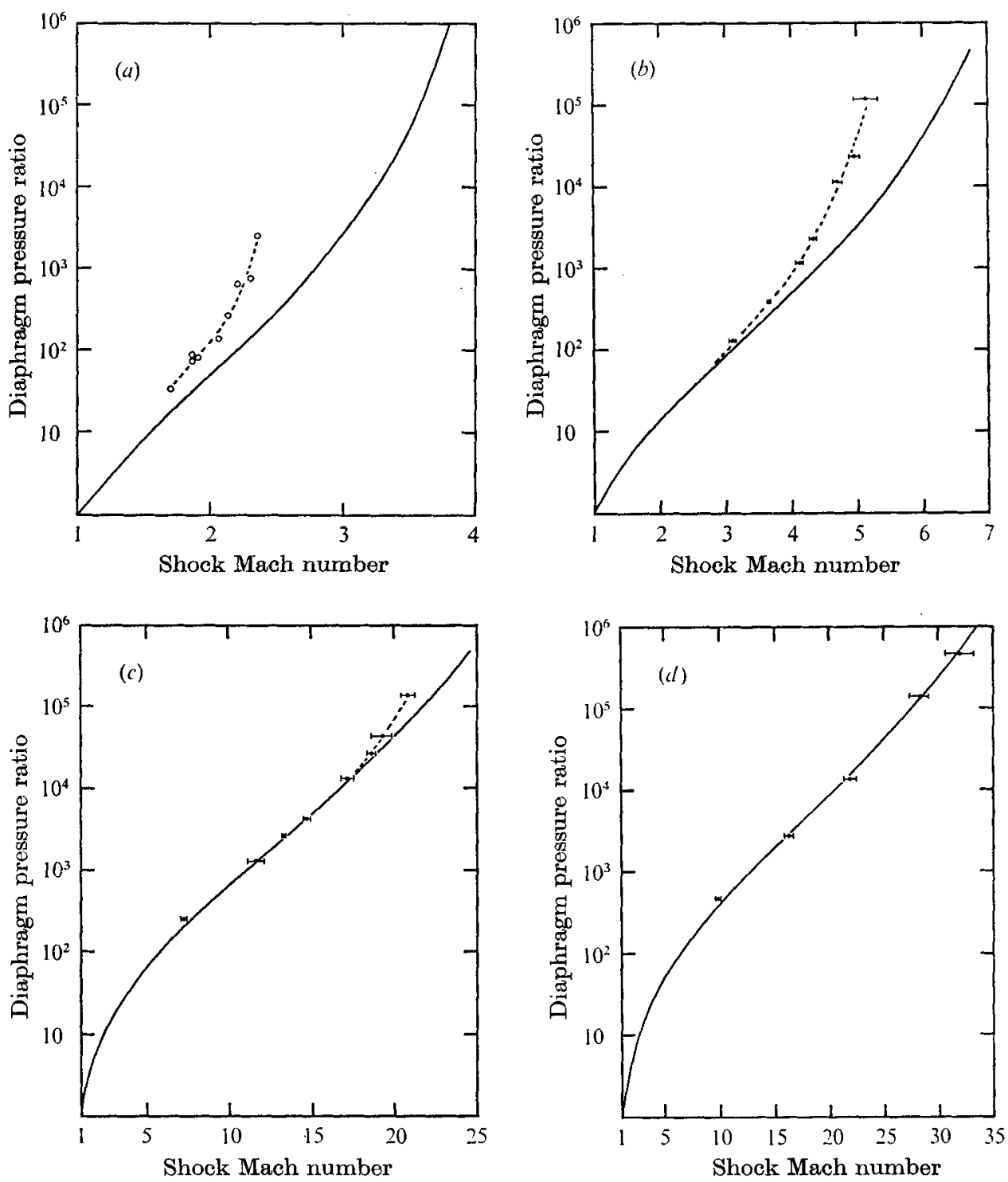

Figure 3. Shock-wave Mach number as a function of diaphragm pressure ratio and test-gas temperature. For all tests, the driver gas and test gas were helium and $T_{4} \doteq 300^{\circ} \mathrm{K}$. - ideal-shock-tube/perfect-gas theory. $(a) T_{1}=300^{\circ} \mathrm{K}$. (b) $T_{1}=77^{\circ} \mathrm{K}$. (c) $T_{1}=4 \cdot 2^{\circ} \mathrm{K}$. (d) $T_{1}=2 \cdot 3^{\circ} \mathrm{K}$.

(actual run data are used for this case). From these results it is important to note several things. The largest Mach number observed at room temperature was $2 \cdot 4$. The increasing departure from ideal values, as the test pressure is decreased, has been observed often before (e.g. by Glass \& Patterson 1955) and has been explained on the basis of boundary-layer effects.

(b) $T_{1}=77^{\circ} \mathrm{K}$. Results for the case in which the coolant was liquid nitrogen are presented in figure $3(b)$ (where the bars correspond to r.m.s. deviations). For 
this case the largest Mach number observed was 5.1. From the figure it seems that the boundary-layer effects are negligible for the case with highest test pressure, 9 Torr, when the shock wave has travelled 20 tube diameters.

(c) $T_{1}=4 \cdot 2^{\circ} \mathrm{K}$. Data for this test-gas temperature are presented in figure $3(c)$. From the figure it is apparent that the trends that were suggested by the $77^{\circ} \mathrm{K}$ data are now more clearly defined. The shock Mach numbers appear to be well predicted by theory. Boundary-layer effects are negligible over this length of test section until the test pressure is below 1 Torr. The largest shock Mach number observed was $20 \cdot 7$.

(d) $T_{1}=2 \cdot 3^{\circ} \mathrm{K}$. Figure $3(d)$ presents the data collected from runs made with $T_{1}=2 \cdot 3^{\circ} \mathrm{K}$. This case represents the culmination of the trends that begin in the $77^{\circ} \mathrm{K}$ case and extend through the $4.2^{\circ} \mathrm{K}$ case. The observed Mach numbers are predicted to within $4 \%$ for all runs made at this temperature. No boundarylayer effects can be seen within the accuracy of the measurements. The highest Mach number observed was $\mathbf{3 2 \cdot 2}$.

(e) $T_{1}=1 \cdot 46^{\circ} \mathrm{K}$. A limited number of runs were made with this test-gas temperature. The observed Mach numbers ranged from 36 to 40 .

\subsection{Shock-tube test times}

As a further characterization of shock-tube performance, test-time data were recorded. The experimental values were determined from the voltage history of the thin-film gauges. One film (located along the leading edge of the glass slide) acted as a stagnation gauge, so a change in the slope of its voltage output could be used to determine the arrival time of the contact surface. For roomtemperature run conditions this arrival time could be clearly seen on the oscillograms, but for the cryogenic cases the change in voltage slope was much less dramatic.

A comparison of the data with theoretical boundary-layer predictions was made difficult by the shortness of the test section (22 tube diameters). For example, the room-temperature data correlated very well with laminar boundarylayer calculations using an 'effective' test-section length that was roughly $50 \%$ of the actual length. At cryogenic temperatures the boundary layer appeared to be laminar for most test pressures and the 'effective' test-section length was $75-100 \%$ of the actual length. $\dagger$

The temperature of the shock-tube wall is important when considering test times for the $4 \cdot 2^{\circ} \mathrm{K}$ and the $2 \cdot 3^{\circ} \mathrm{K}$ cases. If the rise in wall temperature is several degrees, the viscosity of the gas at the wall can increase by a factor of two. If the rise in wall temperature is negligible, the gas behind the shock condenses at the wall for the cases with large test pressures. Either effect decreases the test time.

Using an analysis similar to that of Sturtevant \& Okamura (1969) it was possible to calculate the temperature rise at the wall for a laminar boundary layer. Over the range of test pressures used, the wall temperature was $\sim 8^{\circ} \mathrm{K}$

$\dagger$ A complete comparison of test-time data with theory is presented elsewhere (Cummings 1973). 
for the $4 \cdot 2^{\circ} \mathrm{K}$ cases and $\sim 6^{\circ} \mathrm{K}$ for the $2 \cdot 3^{\circ} \mathrm{K}$ cases. For all run conditions the pressure behind the shock wave was well below the saturated vapour pressure at these temperatures. Hence it may be concluded that the gas did not condense at the walls of the test section. The rise in wall temperature would have been even larger for a turbulent boundary layer.

\section{Conclusions}

A cryogenie shock tube capable of produeing shock waves with large Mach numbers has been developed. The shock-tube performance with regard to the shock velocity appears to be governed by ideal-shock-tube/perfect-gas theory. The conditions in all flow regions can be accurately calculated on the basis of perfect-gas behaviour. Observed test times have been compared with boundarylayer predictions.

The development of this cryogenic shock tube as a practical and economical device leads to questions of modification and application. A cryogenic shock tunnel or Ludwieg tube are possible extensions of the basic shock tube. Using a cryogenically cooled driver section it is possible to use the shock tube as a short-duration wind tunnel to attain extremely large Reynolds numbers.

The cryogenic shock tube is a tool that can be used to investigate fluidmechanical and cryogenic phenomena. Areas of possible application and study are as follows.

(a) Shock waves with large Mach numbers in ideal or real gases.

(b) Flows with large Reynolds numbers.

(c) Phenomena involving the effects of decreased heat capacity and heat conductivity at low temperatures.

(d) Boundary-layer effects due to large ratios of gas/wall temperatures.

(e) Condensation, evaporation, sublimation and critical-point phenomena.

$(f)$ Quantum-mechanical effects at low temperatures.

(g) Use of the rapid increase in pressure and temperature across the shock wave to study chemical reaction rates (e.g. frozen species that are heated with an inert-gas shock), cryogenic materials, superconductors or superfluid helium. $\dagger$

This work was made possible by the U.S. Air Force Office of Scientific Research (Grant AFOSR 71-2092), and is based on part 1 of a thesis submitted in partial fulfilment of the requirements for a $\mathrm{Ph}$.D. degree at the California Institute of Technology. The author wishes to acknowledge the many contributions to this work that were made by Prof. Hans W. Liepmann.

$\dagger$ Results of an experimental investigation of the interaction of shock waves with liquid helium I and II are presented in part 2 of Cummings (1973). 


\section{REFERENCES}

Bennett, F. D. 1957 Aero. Eng. Rev. no. 63.

Cummings, J. C. 1973 Ph.D. thesis, California Institute of Technology.

Glass, I. I. \& Patterson, G. N. 1955 J. Aero. Sci. 22, 73.

Goodyer, M. J. \& Kilgore, R. A. 1972 A.I.A.A. 7th Aero. Testing Conf., Palo Alto, California.

Hrrschferder, J. O., Curtiss, C. F. \& Bird, R. B. 1964 Molecular Theory of Gases and Liquids. Wiley.

Lrepmann, H. W., Cummings, J. C. \& Rupert, V. C. 1973 Phys. Fluids, 16, 332.

Mtrels, H. 1963 Phys. Fluids, 6, 1201.

MtreLs, H. 1964 A.I.A.A. J. 2, 84.

Roshko, A. 1960 Phys. Fluids, 3, 835.

Rupert, V.C. 1972 Ph.D. thesis, California Institute of Technology.

Sturtevant, B. \& Okamura, T. T. 1969 Phys. Fluids, 12, 1723.

Vielde, M. P. 1899 Comptes Rendus Acad. Sci. Paris, 129, 1228.

Warren, W. R. \& Harris, C. J. 1969 Proc. 7th Int. Shock Tube Symp., Toronto.

Yoder, M. J. 1971 Ph.D. thesis, University of Michigan. 